\title{
Tumor suppressor role of microRNA-545 in oral squamous cell carcinoma
}

\author{
GUANGYI YUAN $^{1}$, HAOMING WU ${ }^{1}$, YU DU $^{2}$ and FANGLIN $\mathrm{HE}^{3}$ \\ ${ }^{1}$ Department of Oral and Maxillofacial Surgery, Shunde Hospital of Guangzhou Medical University, Foshan, \\ Guangdong 528315; ${ }^{2}$ Department of Oral and Maxillofacial Surgery, Foshan Chancheng District Xiangyang Hospital, \\ Foshan, Guangdong 52800; ${ }^{3}$ Department of Oral and Maxillofacial Surgery, Zhongshan Xiaolan Renmin Hospital, \\ Zhongshan, Guangdong 528415, P.R. China
}

Received October 18, 2017; Accepted July 20, 2018

DOI: $10.3892 / \mathrm{ol} .2018 .9820$

\begin{abstract}
Oral squamous cell carcinoma (OSCC) is the predominant histological type of oral cancer, and poses an important threat to human health. MicroRNA (miRNA) serves important functions in the pathogenesis of OSCC, and in that of other types of cancer. For example, miR-545 exhibits inhibitory functions in pancreatic ductal adenocarcinoma, lung cancer and hepatocellular carcinoma. In the present study, the function of miR-545 was assessed in OSCC. Obtained results indicated decreased levels of miR-545 in OSCC tissues when comparing with the adjacent normal tissues. Overexpression of miR-545 inhibited HSC4 cells proliferation and migration, and vice versa. Furthermore, miR-545 was identified to target the 3'-UTR of retinoic acid-inducible gene-I (RIG-I), and there was a negative association between RIG-I mRNA expression and miR-545 expression in the 20 OSCC tissues. In conclusion, the present data demonstrate the tumor suppressive role of miR-545 in OSCC.
\end{abstract}

\section{Introduction}

Oral cancer is considered to be part of the head and neck group of cancers. Oral cancer may arise from any part the oral cavity or oropharynx (1). Oral squamous cell carcinoma (OSCC) is the predominant histological type, accounting for $\sim 95 \%$ of oral cancer cases in the USA in 2008 (2). Tobacco smoking, alcohol consumption, poor oral hygiene and human papilloma virus (HPV) infections are the main causes of OSCC $(3,4)$. Conventional oral examination has been the mainstay of oral cancer screening for decades. This

Correspondence to: Dr Fanglin He, Department of Oral and Maxillofacial Surgery, Zhongshan Xiaolan Renmin Hospital, 65 Jucheng Avenue, Zhongshan, Guangdong 528415, P.R. China E-mail: YZhengM_139@126.com

Key words: microRNA-545, oral squamous cell carcinoma, cell proliferation, migration examination is able to detect tumors located in the oral cavity from the early stages, but fails to identify all premalignant oral lesions (5).

Surgery is the primary therapy for oral cancer, especially for patients in advanced stages. Chemotherapy and radiation therapy are often combined with surgical resection to treat patients with advanced oral cancer (6). Nevertheless, the 5-year survival rate is only $\sim 50 \%$ (7). Therefore, the mechanism underlying OSCC development must be elucidated, in order to optimize treatment and improve patient survival.

MicroRNAs (miRNAs) are a type of non-protein coding RNA, conserved in the genomes of animals and plants (8). miRNAs regulate numerous genes post-transcriptionally by binding the 3'-untranslated regions (UTRs) of targeted genes (9). A previous study revealed numerous types of miRNA dysregulation in cancer (10). In OSCC, multiple miRNAs have been identified to be differentially expressed, which indicates that miRNAs may be involved in the pathogenesis of OSCC (11). Previous studies have demonstrated that miR-545 serves inhibitory functions in pancreatic ductal adenocarcinoma (12), lung cancer (13) and hepatocellular carcinoma (14) and the target genes of miR-545 is retinoic acid-inducible gene-I (RIG-I) $(12,14)$ and cyclin D1 and CDK4 (13).

In the present study, the role of miR-545 in OSCC was assessed in vitro and in vivo, aiming to identify the underlying molecular mechanism of the pathogenesis of OSCC. We hypothesize that miR-545 inhibits OSCC by targeting RIG-I.

\section{Materials and methods}

Tissue samples. A total of 20 OSCC tissue samples and their matched adjacent normal tissues were acquired from the Department of Stomatology, Shunde Hospital of Guangzhou Medical University (Foshan, China) from September 2012 to July 2014. Matched adjacent normal tissues were used as controls. The patient information is listed as follows: Median age, 57 years; age range: 41-76 years; sex ratio (M/F): 13/7. The senior pathologists of Shunde Hospital of Guangzhou Medical University confirmed the pathological diagnosis of all OSCC patients. All tissues were frozen immediately and 
preserved in a $-80^{\circ} \mathrm{C}$ freezer for further analysis, including the detection of miR-545 and retinoic acid-inducible gene (RIG)-I mRNA. Written informed consent was obtained from all patients and the present study was approved by the Ethics Committees of Guangzhou Medical University (Guangzhou, China).

Cell culture. Four OSCC cell lines (HSC2, HSC4, SAS, KON) were acquired from the cell bank of the Chinese Academy of Medical Sciences (Shanghai, China). One normal oral keratinocyte cell line (HOK) was purchased from Applied Biological Material (Vancouver, Canada). OSCC lines were cultured in Dulbecco's modified Eagle's medium and Ham's F-12 medium (Sigma-Aldrich; Merck KGaA, Darmstadt, Germany) with $10 \%$ fetal bovine serum (Gibco; Thermo Fisher Scientific, Inc., Waltham, MA, USA). HOK cells were cultured in Prigrow series medium (cat. no. TM4074; Applied Biological Material) with 10\% FBS.

Detection of miR-545 in OSCC tissue samples and cell lines. The levels of miR-545 in 20 OSCC tissue samples and 7 cell lines was detected using reverse transcription-quantitative polymerase chain reaction (RT-qPCR). The total RNA was extracted from each of the 20 specimens using TRIzol ${ }^{\circledR}$ reagent, according to the manufacturer's protocol (Life Technologies; Thermo Fisher Scientific, Inc.). RNA (100 ng) was reverted by ImProm-II TM Reverse Transcription system (Promega Corporation, Madison, WI, USA). Normalization was performed using the $2^{-\Delta \Delta \mathrm{Cq}}$ method (15) using SYBR-Green reagent (Sangon Biotech Co, Ltd., Shanghai, China). The primers for miR-545 were: Primer-Sense, TCA GtAAATGTtTATtAGATGA; Primer-Anti-Sense, GTG CAGGGTCCGAGGTATTC. U6 snRNA was used as the reference gene. The primer of U6 snRNA was listed as follows: Forward, 5'-CTCGCTTCGGCAGCACA-3'; and reverse, 5'-AACGCTTCACGAATTTGCGT-3'. The amplification conditions were: an initial denaturation step at $95^{\circ} \mathrm{C}$ for $10 \mathrm{~min}$, followed by 40 cycles at $95^{\circ} \mathrm{C}$ for $15 \mathrm{sec}$ and $60^{\circ} \mathrm{C}$ for $60 \mathrm{sec}$.

The RIG-I mRNA level in the OSCC tissues was assayed using SYBR-Green reagent (Sangon Biotech Co, Ltd.). The primers for RIG-I (human) were as follows: Forward, 5'-GGACGT GGCAAAACAAATCAG-3' and reverse, 5'-GCAATGTCA ATGCCTTCATCA-3'. The amplification conditions were: $94^{\circ} \mathrm{C}$ for $5 \mathrm{~min} ; 30$ cycles of $94^{\circ} \mathrm{C}$ for $45 \mathrm{sec}, 55^{\circ} \mathrm{C}$ for $45 \mathrm{sec}$ and $72^{\circ} \mathrm{C}$ for $1 \mathrm{~min}$; and $72^{\circ} \mathrm{C}$ for $10 \mathrm{~min}$.

Overexpression and downregulation of miR-545 in OSCC cells. In the OSCC cell lines, miR-545 was overexpressed by miR-545 mimics and decreased by miR-545 antisense oligonucleotides (ASO), as described in a previous study (11). miR-545 mimics, miR-545 ASO and control miRNA were purchased from Sangon Biotech Co, Ltd. miRNAs were transfected into cells using the Lipofectamine 2000 reagent (Invitrogen; Thermo Fisher Scientific, Inc.) according to the manufacturer's protocol. In short, cells were seeded to $70-90 \%$ confluent at transfection, the miR-545 (30 nM) and Lipofectamine 2000 reagent complex were added into cells according to the manufacturer's protocol. Cells were incubated at $37^{\circ} \mathrm{C}$ in a $\mathrm{CO}_{2}$ incubator for $24 \mathrm{~h}$ prior to testing for transgene expression.

Cell proliferation assay. Cellular growth was analyzed using an MTT assay, as previously described (16-20). Briefly, cells were placed into 96 -well plates at a density of $5 \times 10^{3}$ cells/well. The MTT reagent was added into the medium at a final concentration of $0.1 \mathrm{mg} / \mathrm{ml}$, and $100 \mu \mathrm{l}$ of dimethyl sulfoxide was added. The optical density was measured on a microplate reader with a $570 \mathrm{~nm}$ filter.

Cell migration assay. Transwell systems were used to assess cell migration (21). The Transwell chambers $(8.0 \mu \mathrm{m}$ pore size; Sigma-Aldrich; Merck KGaA) were placed in 24-well plates. The miR-545 mimics- or ASO-transfected cells were deprived of FBS for $12 \mathrm{~h}$, and subsequently added to the upper chambers. DMEM Medium containing 10\% FBS was placed in the lower chambers. The cells were incubated in a humidified incubator at $37^{\circ} \mathrm{C}$ for $24 \mathrm{~h}$. Cells in the upper chambers were removed with cotton swabs. The cells attached to the lower surface were fixed in $70 \%$ ethanol for $10 \mathrm{~min}$ at room temperature. The remaining ethanol was removed from the top of the membrane using a cotton-tipped applicator. Cells were then stained with $0.2 \%$ crystal violet into for $10 \mathrm{~min}$ at room temperature. The number of cells that had attached to the lower surface was counted in five randomly selected fields under a microscope (The Eclipse Ti2, Tokyo, Japan) (light, magnification, x200).

Prediction of the putative targets of miR-545. The Targetscan software (http://www.targetscan.org/, accessed September 2017) was used to predict the putative targets of miR-545.

Dual luciferase reporter assays. Cells were seeded at $1 \times 10^{5}$ per well and were serum-starved for $6 \mathrm{~h}$ pre-transfection. Since RIG-I is coded by the DExD/H-Box Helicase 58 (DDX58) gene and there are two miR-545 binding sites on DDX58 (22), the 3'-UTR of RIG-I and mutated controls were cloned and inserted into the reporter plasmid (cat. no. E1761; $500 \mathrm{ng}$ ) and the pGL3-control (cat. no.E1741; 100 ng; Promega Corporation, Madison, WI, USA). miR-545 mimics (50 nM) were then transfected into the HSC4 cells containing the wild-type or mutant 3'-UTR plasmids, using Lipofectamine 2000 (Invitrogen; Thermo Fisher Scientific, Inc.). Cells were harvested, and luciferase activity was measured for each specimen after $24 \mathrm{~h}$ using the Dual-Luciferase Reporter assay system (cat. no. E1910; Promega Corporation). Mutants of RIG-I 3'-UTR were generated using the Site-Directed Mutagenesis kit (Thermo Fisher Scientific, Inc.).

Western blotting analysis. Cells were frozen and lysed in lysis buffer $(150 \mathrm{mM} \mathrm{NaCl}, 50 \mathrm{mM}$ Tris-HCI, $1 \%$ Triton X-100 and $0.1 \%$ SDS) with a Protease Inhibitor Cocktail (cat. no. S8820; Sigma-Aldrich; Merck KGaA, Darmstadt, Germany) and a Phosphatase Inhibitor (cat. no. P0044; Sigma-Aldrich). For RIG-I analysis, a RIG-I antibody (ab132505) was used at a dilution of 1:1,000 and incubated at $4^{\circ} \mathrm{C}$, overnight), followed by detection with a peroxidase-linked antibody (ab6759; both Abcam, Cambridge, UK) to rabbit antibody IgG of 1:2,000 


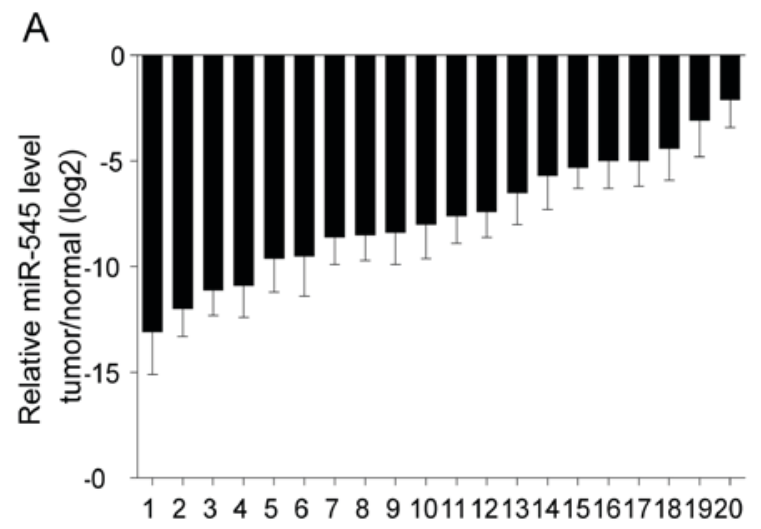

C

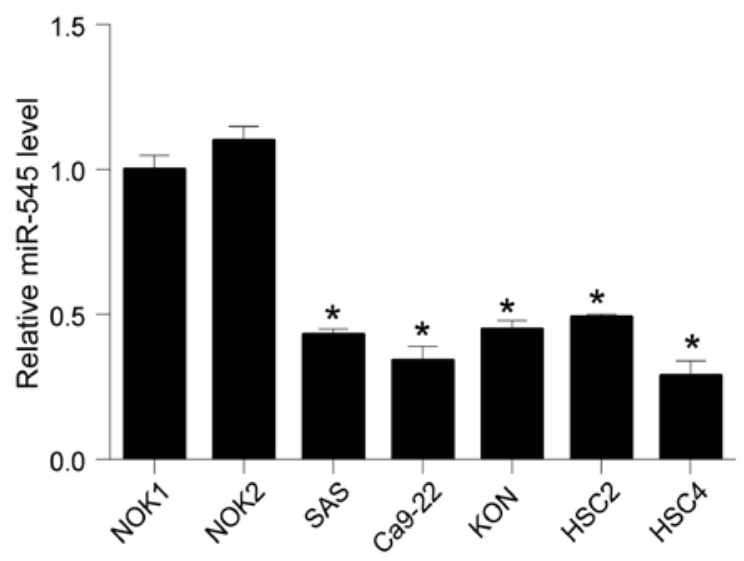

B

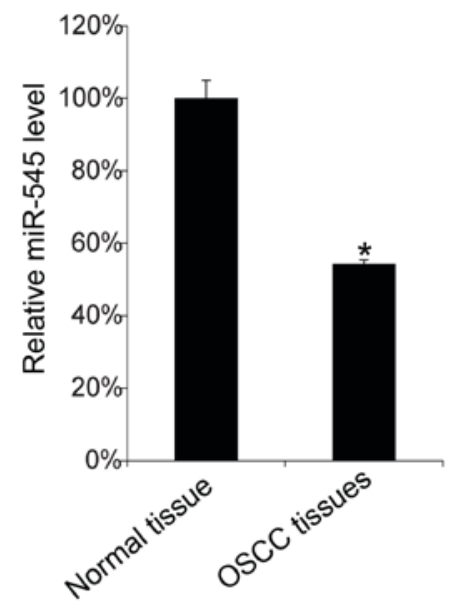

Figure 1. Low level of miR-545 in OSCC tissue samples. The miR-545 levels of 20 OSCC tissues and their matched adjacent normal tissues were assessed by RT-qPCR. (A) The relative miR-545 levels tumor/normal $(\log 2)$ were listed. (B) The mean miR-545 values of the OSCC tissues and their matched adjacent normal tissues were also recorded. (C) The miR-545 levels of 4 OSCC cell lines (HSC2, HSC4, SAS, KON) and normal oral keratinocyte cells (HOK) were assayed by RT-qPCR. These experiments were performed in triplicate ${ }^{*} \mathrm{P}<0.05$. OSCC, oral squamous cell carcinoma; RT-qPCR, reverse transcription-quantitative polymerase chain reaction; $\mathrm{miR}$, microRNA.

dilution incubated at room temperature for $2 \mathrm{~h}$. Proteins were detected using an Enhance Chemiluminesence Western Blotting Detection Reagent (GE Healthcare, Chicago, IL, USA). Images were analyzed using Image J (National Institutes of Health, Bethesda, MD, USA).

Statistical analysis. All experiments were repeated three times. Data are presented as the mean \pm the standard deviation. A two-tailed Student's t-test was used to analyze the mean value between two groups. One way Analysis of variance was used to test the mean value among three groups or more with post hoc contrasts by Student-Newman-Keuls test. The correlation between miR-545 and RIG-I levels were examined by Pearson correlation coefficient analysis. $\mathrm{P}<0.05$ was considered to indicate a statistically significant difference. All calculations were performed using SPSS software (version 16.0; SPSS, Inc., Chicago, IL, USA).

\section{Results}

miR-545 levels in OSCC tissues. To understand the function of miR-545, the miR-545 levels in 20 OSCC tissue samples and their matching adjacent normal tissues were evaluated using RT-qPCR. Overall, the analysis revealed a decreased level of miR-545 in each tumor tissue compared with the matched normal tissues (Fig. 1A). The mean level of miR-545 in the 20 OSCC samples was calculated and compared to the mean level of miR-545 in the normal tissues. As presented in Fig. 1B, the mean level of miR-545 obtained for the 20 OSCC tissues was decreased compared with the mean in normal tissues $(\mathrm{P}<0.05)$. Similarly, a subsequent comparison revealed that miR-545 was more abundant in normal oral keratinocyte cell lines (HOK) than in four OSCC cell lines (HSC2, HSC4, SAS, KON; Fig. 1C) (the mean value in NOK1 or NOK2 were compared with the four OSCC cell line separately, $\mathrm{P}<0.05$ ).

The in vitro role of miR-545 in OSCC cells. As HSC4 cells exhibited relatively lower miR-545 levels when compared with the other examined OSCC cell lines, the HSC4 cells were selected for further investigation. The miR-545 levels were regulated using miR-545 mimics and miR-545 ASO transfections, which upregulated and downregulated miR-545, respectively. miR-545 mimics transfection increased the level of miR-545 in HSC4 cells, whereas miR-545 ASO transfection resulted in a decrease (Fig. 2A) $(\mathrm{P}<0.05)$. Additionally, cell growth was assessed following transfection. As presented in Fig. 2B, the upregulation of miR-545 inhibited cell growth, whereas its downregulation promoted cell growth in HSC4 cells. Subsequently, cell migration 
was investigated following transfection, and it was observed that miR-545 mimics decrease the number of migrating cells, whereas miR-545 ASO transfection results in an increase in cell migration in HSC4 cells (Fig. 2C) $(\mathrm{P}<0.05)$.

RIG-I is a target gene of miR-545. To understand the molecular mechanism of miR-545 in OSCC, its potential target gene was investigated. It has been reported that the 3'-UTR of RIG-I may be targeted by miR-545 in pancreatic ductal adenocarcinoma (12). RIG-I is localized in the cytosol, where it recognizes the 5'-triphosphate RNA (3p-RNA) generated by viral RNA polymerases $(22,23)$. In the present study, the association between miR-545 and RIG-I in OSCC was investigated. The binding sites were mutated as described in a previous study (Fig. 3A) (12). The mutated sites were cloned into a luciferase reporter plasmid. miR-545 mimics and the reporter plasmid were co-transfected into HSC4 cells. The activity of luciferase was assessed $12 \mathrm{~h}$ after transfection. The upregulation of miR-545 inhibited luciferase activity $(\mathrm{P}<0.05)$, whereas the mutation of binding site 1 and site 2 partly restored it, indicating that miR-545 targets RIG-I in HSC4 cells (Fig. 3B). Subsequently, the RIG-I protein levels following miR-545 mimics transfection were determined, and it was identified that miR-545 transfection inhibited the RIG-I protein levels in HSC4 cells (Fig. 3C). Furthermore, the RIG-I mRNA expression levels were assessed in 20 OSCC tissues, and it was identified that RIG-I mRNA expression was associated with miR-545 expression. The results obtained revealed a negative association between RIG-I mRNA expression and miR-545 expression in the 20 OSCC samples (Fig. 3D) $(\mathrm{P}<0.05)$.

\section{Discussion}

In the present study, the function of miR-545 in OSCC was investigated. The present data revealed that miR-545 levels in OSCC tissues were lower than levels in matched adjacent normal tissues. Overexpression of miR-545 inhibited HSC4 proliferation and migration, and vice versa. The potential target gene of miR-545 was identified to be RIG-I.

The antitumor role of miR-545 have been confirmed in other types of cancer, including lung cancer (13), pancreatic ductal adenocarcinoma (12), and epithelial ovarian cancer (23). To the best of our knowledge, there are no reports pertaining to the oncogenic function of miR-545. Thus, we hypothesize that miR-545 exhibited an antitumor function in various types of cancer.

The present data demonstrated the inhibitory role of miR-545 in OSCC. Furthermore, a negative association between RIG-I mRNA expression and miR-545 expression was observed in the OSCC tissues. To our knowledge, this is the first study to highlight the function of miR-545 and RIG-I in OSCC. Notably, RIG-I is a part of the key pathway of human papilloma virus (HPV) infection. Non-self dsDNA of HPV may serve as template for transcription and induce type I interferon and nuclear factor- $\kappa-B$ through the RIG-I pathway (24-26). Additionally, HPV infection was an independent factor associated with OSCC after adjusting for age, smoking and alcohol use (27). Thus, it is hypothesized that HPV may exploit the regulation mechanism of miR-545 and RIG-I in the pathogenesis of OSCC. The present study identified that miR-545 was
A

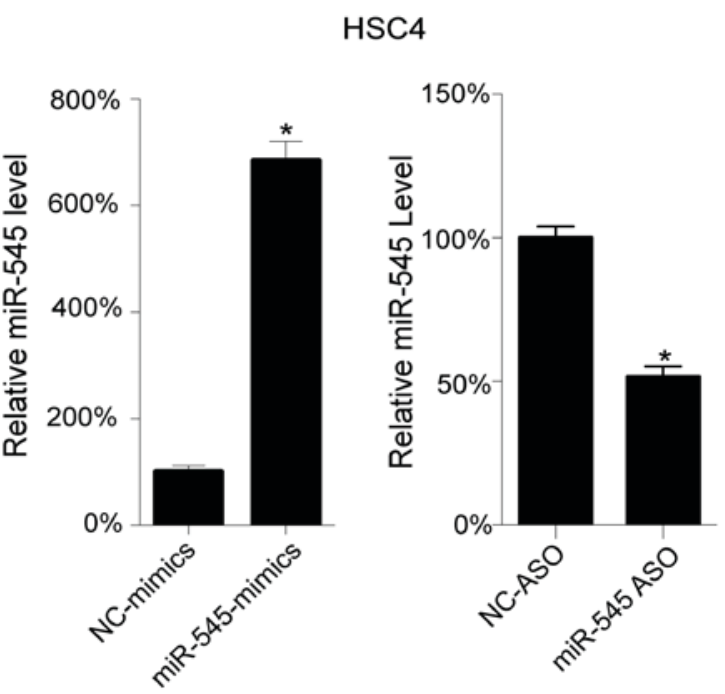

B

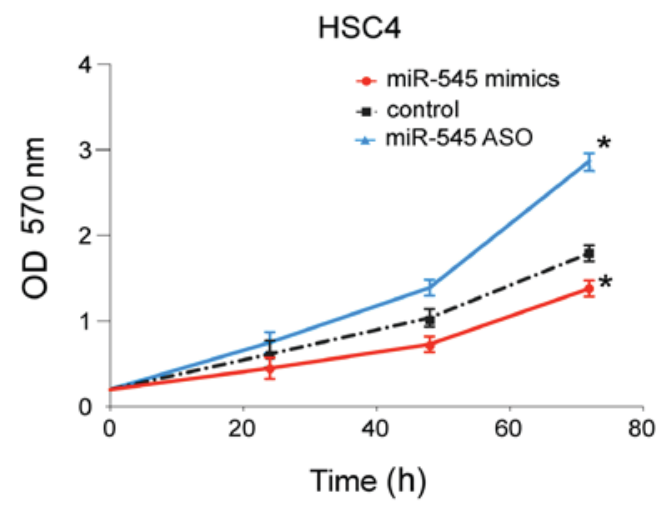

C

HSC4

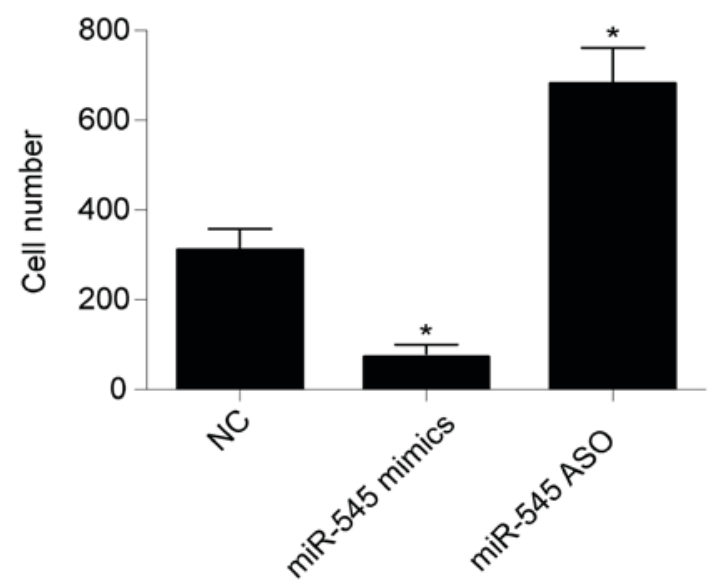

Figure 2. Overexpression of miR-545 inhibited HSC4 proliferation and migration, and vice versa. (A) In HSC4 cells, miR-545 was overexpressed by miR-545 mimics transfection, and downregulated by miR-545 ASO transfection. (B) The cellular proliferation of HSC4 cells, following transfection, was assayed by MTT analysis. (C) The migrated number of cells following cellular migration analysis. These experiments were performed in triplicate. ${ }^{*} \mathrm{P}<0.05$. ASO, antisense oligonucleotides; NC, negative control; OD, optical density; miR, microRNA.

able to target RIG-I in OSCC cells. Besides RIG-I, miR-545 has been demonstrated to target cyclin D1 and CDK1 in lung cancer (13), implying that miR-545 has multiple targets in 
A

$$
\begin{array}{r}
\text { Position 563-570 of DDX58 3' UTR } \\
\text { hsa-miR-545 } \\
\text { Position 1074-1081 of DDX58 3' UTR } \\
\text { hsa-miR-545 }
\end{array}
$$

Mutant site 1 of position 563-570 of DDX58 3' UTR

hsa-miR-545

Mutant site 2 of position 1074-1081 of DDX58 3' UTR

hsa-miR-545

\section{5' ...UAUUGAGUCCUCCUCUUUGCUGA... \\ | | | | | | | \\ 3' CGUGuguUAUUUACAAACGACU}

5' ...UGuCACUUAGCAAGguUUGCUGA...

| | | | | । |

3' CGUGUGUUAUUUACAAACGACU

5' ...UAUUGAGUCCUCCUCUUAAAAGA...

3' CguguguUAUUUACAAACGACU

5' ...UGUCACUUAGCAAGGUUAAAAGA...

3' CGUGUGUUAUUUACAAACGACU

B HSC4

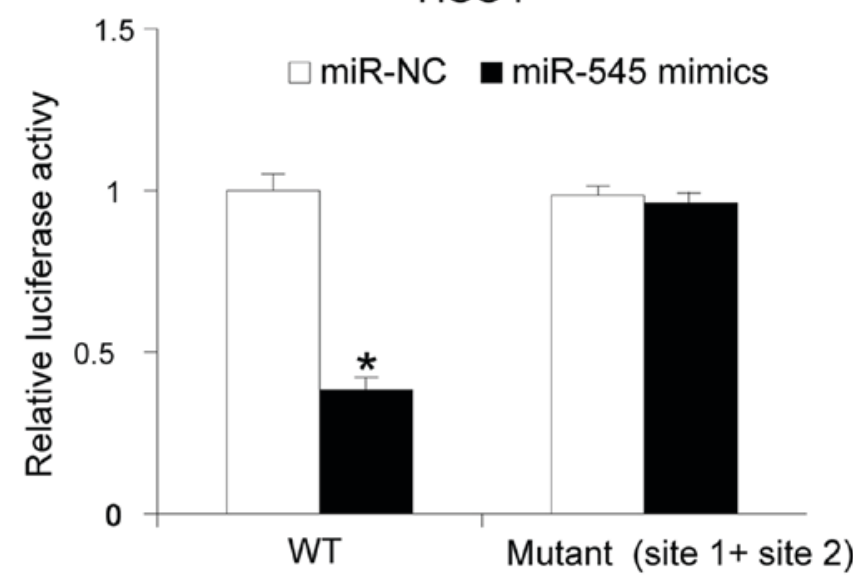

C

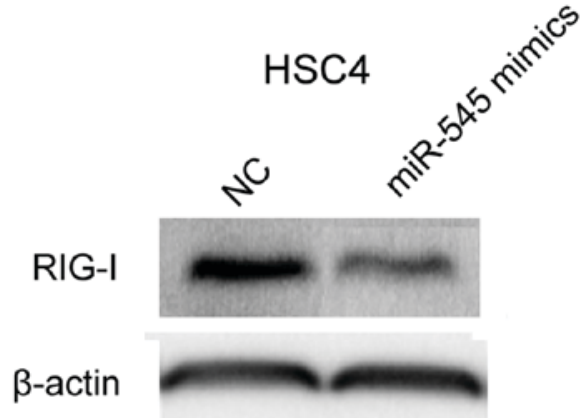

D

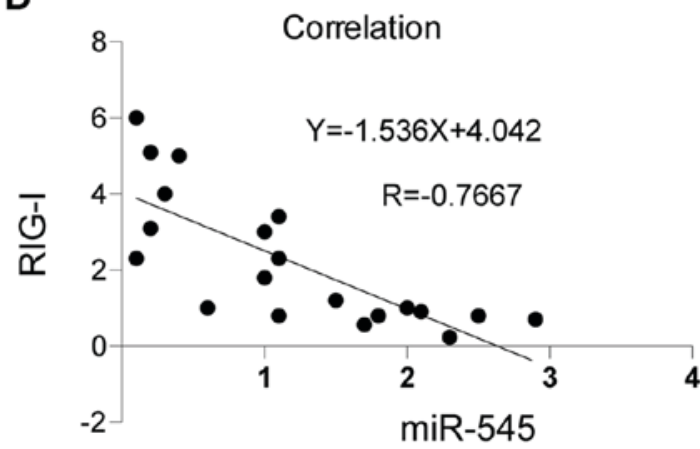

Figure 3. RIG-I is targeted by miR-545. (A) The binding sites and the location of the two mutations (position 563-570 and position 1074-1081) were listed. (B) miR-545 mimics and a plasmid containing either wild-type or mutated 3'-UTR sequences of RIG-I were transfected into HSC4 cells and, 48 h later, luciferase activity was analyzed. (C) miR-545 mimics were transfected into HSC4 cells and the amount of RIG-I protein was determined by western blotting. (D) The correlation between miR-545 and RIG-I levels were examined by Pearson correlation coefficient analysis. Each experiment was repeated at least three times. ${ }^{*} \mathrm{P}<0.05$ vs. miR-545 mimics. miR, microRNA; UTR, untranslated region; DDX58, gene encoding RIG-I; RIG-I, retinoic acid-inducible gene-I.

cancer. In conclusion, the present data suggest an inhibitory role of miR-545 in OSCC.

\section{Acknowledgements}

The authors would like to thank Mr. Li Dayin (Department of Oral and Maxillofacial Surgery, Shunde Hospital of Guangzhou Medical University) for technical assistance.

\section{Funding}

No funding was received.

\section{Availability of data and materials}

All data generated or analyzed during this study are included in this published article. 


\section{Authors' contributions}

GY collected patient data and performed cell experiments. HW and YD performed PCR, western blotting and other molecular experiments. FH contributed to study design and manuscript writing.

\section{Ethics approval and consent to participate}

Written informed consent was obtained from all patients, and the Ethics Committees of Guangzhou Medical University approved the present study.

\section{Patient consent for publication}

All patients have provided their consent for the use of their information and samples for scientific research and publication.

\section{Competing interests}

The authors declare that they have no competing interests.

\section{References}

1. Rivera C: Essentials of oral cancer. Int J Clin Exp Pathol 8: 11884-11894, 2015.

2. Choi S and Myers JN: Molecular pathogenesis of oral squamous cell carcinoma: Implications for therapy. J Dent Res 87: 14-32, 2008.

3. Gupta PC: Mouth cancer in India: A new epidemic? Indian Med Assoc 97: 370-373, 1999.

4. Mehrotra R, Singh M, Kumar D, Pandey AN, Gupta RK and Sinha US: Age specific incidence rate and pathological spectrum of oral cancer in Allahabad. Indian J Med Sci 57: 400-404, 2003

5. Lingen MW, Kalmar JR, Karrison T and Speight PM: Critical evaluation of diagnostic aids for the detection of oral cancer. Oral Oncol 44: 10-22, 2008

6. O'neill V and Twelves C: Oral cancer treatment: Developments in chemotherapy and beyond. Br J Cancer 87: 933-937, 2002.

7. Ries LAG, Melbert D, Krapcho M, Stinchcomb DG, Howlader N, Horner MJ, Mariotto A, Miller BA, Feuer EJ and Altekruse SF: SEER Cancer Statistics Review, 1975-2005 (Based on November 2007 SEER data submission). National Cancer Institute, Bethesda, MD, 2008.

8. Du T and Zamore PD: microPrimer: The biogenesis and function of microRNA. Development 132: 4645-4652, 2005.

9. Bartel DP: MicroRNAs: Target recognition and regulatory functions. Cell 136: 215-233, 2009.

10. Farazi TA, Hoell JI, Morozov P and Tuschl T: MicroRNAs in human cancer. Adv Exp Med Biol. 1-774: 1-20, 2013.

11. Manikandan M,Rao AKDM,Arunkumar G, Manickavasagam M, Rajkumar KS, Rajaraman R and Munirajan AK: Oral squamous cell carcinoma: microRNA expression profiling and integrative analyses for elucidation of tumourigenesis mechanism. Mol Cancer 15: 28, 2016.
12. Song B, Ji W, Guo S, Liu A, Jing W, Shao C, Li G and Jin G: miR-545 inhibited pancreatic ductal adenocarcinoma growth by targeting RIG-I. FEBS Lett 588: 4375-4381, 2014.

13. Du B, Wang Z, Zhang X, Feng S, Wang G, He J and Zhang B MicroRNA-545 suppresses cell proliferation by targeting cyclin D1 and CDK4 in lung cancer cells. PLoS One 9: e88022, 2014.

14. Liu Z, Dou C, Yao B, Xu M, Ding L, Wang Y, Jia Y, Li Q, Zhang $\mathrm{H}$, Tu K, et al: Ftx non coding RNA-derived miR-545 promotes cell proliferation by targeting RIG-I in hepatocellular carcinoma. Oncotarget 7: 25350-25365, 2016.

15. Livak KJ and Schmittgen TD: Analysis of relative gene expression data using real-time quantitative PCR and the 2(-Delta Delta C(T)) method. Methods 25: 402-408, 2001.

16. Mosmann T: Rapid colorimetric assay for cellular growth and survival: Application to proliferation and cytotoxicity assays. J Immuno Methods 65: 55-63, 1983.

17. Roehm NW, Rodgers GH, Hatfield SM and Glasebrook AL: An improved colorimetric assay for cell proliferation and viability utilizing the tetrazolium salt XTT. J Immunol Methods 142: 257-265, 1991.

18. Gerlier D and Thomasset N: Use of MTT colorimetric assay to measure cell activation. J Immunol Methods 94: 57-63, 1986.

19. Berridge MV, Tan AS, McCoy KD and Wang R: The biochemical and cellular basis of cell proliferation assays that use tetrazolium salts. Biochemica 4: 14-19, 1996.

20. Weichert H, Blechschmidt I, Schröder S and Ambrosius H: The MTT-assay as a rapid test for cell proliferation and cell killing: Application to human peripheral blood lymphocytes (PBL). Allergie Immunol (Leipz) 37: 139-144, 1991.

21. Rüster B, Grace B, Seitz O, Seifried E and Henschler R: Induction and detection of human mesenchymal stem cell migration in the 48-well reusable transwell assay. Stem Cells Dev 14: 231-235, 2005.

22. Hornung V, Ellegast J, Kim S, Brzózka K, Jung A, Kato H, Poeck H, Akira S, Conzelmann KK, Schlee M, et al: 5'-Triphosphate RNA is the ligand for RIG-I. Science 314: 994-997, 2006.

23. Jia X, Liu X, Li M, Zeng Y, Feng Z, Su X, Huang Y, Chen M and Yang X: Potential tumor suppressing role of microRNA-545 in epithelial ovarian cancer. Oncol Lett 15: 6386-6392, 2018.

24. Pichlmair A, Schulz O, Tan CP, Näslund TI, Liljeström P, Weber F and Reis e Sousa C: RIG-I-mediated antiviral responses to single-stranded RNA bearing 5'-phosphates. Science 314: 997-1001, 2006.

25. Chiu YH, Macmillan JB and Chen ZJ: RNA polymerase III detects cytosolic DNA and induces type I interferons through the RIG-I pathway. Cell 138: 576-591, 2009.

26. Ablasser A, Bauernfeind F, Hartmann G, Latz E, Fitzgerald KA and Hornung V: RIG-I-dependent sensing of poly $(\mathrm{dA}: \mathrm{dT})$ through the induction of an RNA polymerase III-transcribed RNA intermediate. Nat Immunol 10: 1065-1072, 2009.

27. Gan LL, Zhang H, Guo JH and Fan MW: Prevalence of human papillomavirus infection in oral squamous cell carcinoma: A case-control study in Wuhan, China. Asian Pac J Cancer Prev 15: 5861-5865, 2014.

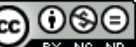

This work is licensed under a Creative Commons Attribution-NonCommercial-NoDerivatives 4.0 International (CC BY-NC-ND 4.0) License. 